\title{
EFEITO DA PUNICA GRANATUM (ROMÃ) EM PROCESSOS INFLAMATÓRIOS INDUZIDOS EM RATOS E SUAS IMPLICAÇÕES NO EIXO HIPOTÁLAMO-HIPÓFISE- ADRENAL
}

\section{Effect of Punica granatum (pomegranate) on induced inflammatory in rats and it's implications on hypothalamus-hypophysis-adrenal axis}

Katienne Brito Marcelino 1,2 , Nilson Afonso da Silva Júnior², Ana Gomes Freitas²,
Cristiane Chaves Campos², Wataro Nelson Ogawa

\section{RESUMO}

Após injúria tissular iniciam-se os eventos da cicatrização: inflamação, proliferação e remodelação. A pesquisa teve como objetivo avaliar o efeito fitoterápico da romã em feridas e suas implicações nas vias neuroendócrinas do estresse. Protocolo CEUA 02/2017. Induziu-se feridas nos dorsos de ratos anestesiados. Tratados por 3 dias com salina ou romã (chá e/ou polpa). Ao final do $4^{\circ}$ dia, foi inoculado Azul de Evans (AE, 0,3 $\mathrm{mL} 2,5 \%$ ) em todos os ratos, pela veia hepática. Uma das metades das lesões, após 24 horas, teve o AE lido no comprimento de onda $640 \mathrm{~nm}$ e a outra metade foi novamente pesada a $60^{\circ} \mathrm{C}$ a diferença expressa como exsudato. As adrenais participaram da determinação do conteúdo de ácido ascórbico (AAA), leituras em $520 \mathrm{~nm}$. ANOVATukey com significância de 5\%. Encontrado aumento significativo de AAA, peso das adrenais e permeabilidade ao $\mathrm{AE}$ diminuídos, nos tratados com romã, comparado ao grupo com salina $(F=5,8 ; P=0,005 ; F=5,9 ; P<0,01 ; F=9,8 ; P=0,0011)$ o que sugere ação anti-inflamatória e propriedade adaptógena da romã, embora o conteúdo médio de exsudato tenha sido igual em todas as condições de tratamento $(F=2,7 ; P=0,0677)$.

Palavras-chave: Permeabilidade capilar. Punicaceae. Inflamação. Estresse.

\section{ABSTRACT}

After tissular injury initiate the cicatrization events: inflammation, proliferation and reshuffle. The research had as objectives evaluate the herbal remedy effects of pomegranate on wounds and it's implications in neuroendocrine pathways of stress. Protocolo CEUA 02/2017. Induced wounds on anesthetized rats backs. Treated for 3 days with saline or pomegranate (tea and/or pulp). At the end of fourth day, was inoculated Evans blue (EB, $0,3 \mathrm{~mL} 2,5 \%$ ) by hepatic vein in all rats. One half of the lesions, after 24 hours, had read the EB at $640 \mathrm{~nm}$ wave-length and the other half was weighted at $60^{\circ} \mathrm{C}$, diference expressed as exudate. The adrenals participate at the content determination of ascorbic avid (AAA), readings at 520 nem. ANOVA-Turkey significance of $5 \%$. Found a significant increased of $A A A$, adrenals weight and permeability to $A E$ decreased at those treated with pomegranate compared to the group with saline $(F=5,8 ; P=0,005 ; F=5,9 ; P<0,01 ; F=9,8 ; P=0,0011)$ what suggests the pomegranate anti-inflammatory action and adaptogenic property, although the exudate medium content had been equal in all treatment conditions $(F=2,7 ; P=0,0677)$.
${ }^{1}$ Graduada em Enfermagem pela Universidade do Estado da Bahia (UNEB)

E-mail:

katmarcelino@hotmail.com..

${ }^{2}$ Acadêmico do curso de Medicina da Universidade de Gurupi UnirG, Gurupi-TO

${ }^{3}$ Mestre em Fisiologia Médica e Doutor em Ciências pela Faculdade de Medicina de Ribeirão Preto - USP, Docente Titular-3 do curso de Medicina da Universidade de Gurupi - UnirG, Gurupi-TO.

Keywords: Capillary Permittivity. Punicaceae. Inflammation. Stress. 


\section{INTRODUÇAOO}

As plantas medicinais vêm sendo utilizadas popularmente desde os primórdios da civilização, com diversas finalidades, principalmente para a cura e a prevenção de doenças. Esse conhecimento, passado oralmente através de gerações, é uma importante fonte para a descoberta de novos princípios ativos (SANTOS et al., 2011).

A flora brasileira é considerada diversificada apresentando inúmeras espécies com potencial medicinal. A grande parte dos princípios ativos de importância farmacológica encontrada nos extratos vegetais, de modo geral, é oriunda de uma variedade de metabólitos secundários que possuem uma constituição complexa, alcançando alvos terapêuticos nas doenças humanas e que são produzidos para modular seus próprios metabolismos (FERREIRA e PINTO, 2010).

Entre as espécies vegetais presentes no território brasileiro, ressalta-se a Punica granatum L., planta arbustiva e frutífera utilizada com finalidade ornamental e por propriedades terapêuticas cicatrizante, antioxidante, antimicrobiana e anti-inflamatória que Ihe são atribuídas pela cultura popular. Partes desse vegetal são utilizadas pela população de forma direta (através de infusões com a casca do fruto) ou em produtos naturais comercializados pelo país. Com isso, tem despertado o interesse de pesquisadores em investigar tais e outras possíveis características e ratificar o seu uso (DEGASPARI et al., 2011; TAKATA et al., 2014; TRINDADE et al., 2009).

Das plantas com características fitoterápicas, há um grupo classificado como adaptógenos. Para Carlini e Mendes (2011), os adaptógenos são substâncias com a capacidade de intensificar a resistência do organismo diante de agentes estressores diversos e com isso promoverem melhor adaptação dos organismos às situações incomuns. Diante das características medicinais, a possibilidade de a romã ser uma planta adaptógena deve ser pesquisada, uma vez que assim sendo, poderia ser utilizada cronicamente em favor da manutenção e da homeostase do organismo. Segundo Davydov e Krikorian (2000), algumas plantas adaptógenas possuem componentes com estruturas moleculares análogas, por exemplo, à de glicocorticoides e de mineralocorticoides, assim poderiam mimetizar a ação desses hormônios esteroidais contribuindo para a atenuação de processos inflamatórios.

Dentre os estudos já publicados, Nascimento Júnior et al (2015) realizou seu trabalho com extrato da romã aplicado em cavidade oral de ratos Wistar e, por método histológico, evidenciou que a romã (Punica granatum L.) possui uma ação cicatrizante nas 
mucosas orais e Tozetto et al (2017) demonstrou uma atividade antioxidante da romã sinalizando um potencial uso comercial de várias apresentações da planta no combate ao estresse oxidativo.

Os resultados obtidos por Argenta et al (2012) mostra uma atividade inibitória in vitro de colônias bacterianas, bem como os obtidos por Trindade et al (2009) que identificou uma atividade antimicrobiana em Gram-positivos provinda da tintura das cascas do fruto da Romã. O uso do extrato da casca do $P$. granatum $L$., por sua vez, parece mostrar resultados satisfatórios em casos de câncer de cólon induzido por via intrarretal com $\mathrm{N}$ metilnitrosoureia em ratos Sprague-Dawley, onde os estudos experimentais de apoptose mostrou resultados de atenuação da inflamação e suprimiu a proliferação de células tumorais (AHMED et al., 2017).

Apesar do fruto da romã ser utilizado popularmente em larga escala, na literatura científica há lacunas no que se refere à comprovação de sua eficácia terapêutica sob o enfoque de mecanismos de ação ainda não inteiramente esclarecidos.

A presente pesquisa se justifica no intuito da tentativa de adicionar e ampliar os achados aos demais já existentes acerca da eficácia da ação anti-inflamatória da fruta, utilizando uma abordagem ainda não empregada nas linhas de pesquisa tradicionais. Os métodos empregados para validar o uso terapêutico da romã seguem basicamente as técnicas histológicas, sendo que neste estudo foi utilizada a mensuração da permeabilidade ao Azul de Evans em processos inflamatórios utilizando um método espectrofotométrico e também de investigar se a romã possui uma propriedade adaptógena, através da mensuração do conteúdo de ácido ascórbico da adrenal.

Tomando as considerações feitas acima como ponto de partida, o presente estudo teve como objetivo avaliar a ação anti-inflamatória da romã em feridas abertas induzidos por lesões experimentais na região dorso-costal de ratos e investigar as suas implicações nas vias neuroendócrinas do estresse.

\section{MATERIAIS E METODOS}

O estudo foi realizado no Laboratório de Fisiologia da Universidade de Gurupi, UnirG, Gurupi-TO. Foram utilizados 24 ratos sem distinção de gênero (linhagem Wistar albinos), provenientes do Biotério da UnirG. Animais adultos e clinicamente saudáveis, pesando de 300-400 gramas e idade aproximada de três meses, foram mantidos no laboratório em caixas de polipropileno $(41 \times 34 \times 16 \mathrm{~cm})$ forradas com maravalha depositados em estantes 
de metal, caixas higienizadas a cada dois dias, com cinco animais/caixa, climatizado em torno de $27^{\circ} \mathrm{C}$ e ciclo circadiano obedecido (claro/escuro com períodos alternados de 12 horas), com um mínimo de manipulação a não ser o estritamente necessário e fornecimento de água e ração ad libitum. Durante os quatro dias de experimentação, os ratos foram subdivididos em quatro grupos de acordo com os tratamentos recebidos em seus ferimentos: grupos: G1 (salina 0,9\%, 5 ratos), G2 (chá, 7 ratos), G3 (polpa, 5 ratos) e G4 (chá+polpa, 7 ratos). Outros seis animais foram usados em estudos-piloto realizados previamente, sendo que não entraram no cômputo dos resultados finais servindo apenas para ajustes das técnicas utilizadas.

O trabalho foi previamente aprovado pela Comissão de Ética na Utilização de Animais (CEUA- UnirG), sob o protocolo número 02/2017.

\subsection{PREPARAÇÕES DA POLPA E DO CHÁ}

Foi utilizado o extrato da polpa e do chá das cascas dos frutos de P. granatum. Inicialmente os frutos foram lavados, secos e em seguida abertos, sendo a polpa separada das sementes do fruto. O extrato da polpa foi obtido por maceração e coagem e guardado a temperatura de $6 \stackrel{\circ}{\mathrm{C}}$ até o momento da administração. As cascas da Romã foram separadas e secadas em estufa por 48 horas a temperatura de $50 \stackrel{\circ}{C}$, em seguida moídas em um moedor elétrico e armazenadas em sacos plásticos hermeticamente fechados em temperatura ambiente até o dia das aplicações. Quantidade de 20 gramas foram colocados em infusão em $100 \mathrm{~mL}$ de água destilada a $100{ }^{\circ} \mathrm{C}$ por 7 minutos, coado e armazenado a $6 \stackrel{\circ}{\circ}$. O extrato do fruto, o chá e o extrato mais o chá, foram administrados nos ferimentos com conta-gotas (5 gotas), uma vez ao dia durante 3 dias.

\subsection{PROCEDIMENTO EXPERIMENTAL}

Todos os ratos foram anestesiados com tiopental sódico $(50 \mathrm{mg} / \mathrm{kg}$, ThiopentaxR, Cristália) e induzidos os processos inflamatórios oriundos de feridas superficiais produzidos nos dorsos por um tubo cilíndrico de aço de base em formato de bisel circular de raio $1 \mathrm{~cm}$. A pele circular foi delicadamente retirada com auxílio de pinça e tesoura de ponta fina sob foco luminoso de uma lente de aumento 8 x. O grupo controle-G1 foi tratado diariamente durante 3 dias com 5 gotas de salina a 0,9\%. Os grupos experimentais foram tratados diariamente, durante 3 dias, respectivamente, com 5 gotas de chá de romã (grupo G2), 5 gotas de polpa de romã (grupo G3) e 5 gotas da mistura chá + romã (grupo G4). 
Ao final do 40 dia, todos os ratos foram anestesiados e, após incisão abdominal e abertura em véu, foram inoculados pela veia hepática $0,3 \mathrm{~mL}$ de Azul de Evans (AE) 2,5\% ( $\mathrm{AE}$, marca Vetec). Após 15 minutos, as regiões injuriadas pelo processo inflamatório foram excisadas, cortadas ao meio, pesadas e, em uma metade o AE foi extraído com $4 \mathrm{~mL}$ de formamida (Vetec) em tubo de ensaio. Decorridos 24 horas, leituras espectrofotométricas dos sobrenadantes dos tubos foram realizadas no espectrofotômetro (marca Kasuaki®), no comprimento de onda fixado em $640 \mathrm{~nm}$ e as absorbâncias transformadas em quantidades por uma curva padrão de $A E$. A outra metade da ferida excisada foi pesada em balança analítica e novamente pesada após 24 horas em estufa a 60 0C. A diferença de peso foi considerada como quantidade de exsudação do processo inflamatório, expresso em porcentual relativo. Todos os animais foram sacrificados na vigência da anestesia com injeção de $0,2 \mathrm{~mL}$ de $\mathrm{KCl} 1 \mathrm{M}$ no coração após esternotomia e perfuração diafragmática.

Durante a inoculação do $A E$ pela via porta hepática, a adrenal esquerda foi rapidamente excisada, dissecada e pesada numa balança analítica com 3 dígitos (Bel Equipamentos Ltda). A adrenal foi macerada com $10 \mathrm{~mL}$ de ácido metafosfórico $(2,5 \%$, Metaquímica) e, em seguida o extrato filtrado (papel de filtro qualitativo, Prolab). Um volume de $2 \mathrm{~mL}$ do filtrado foi coletado com pipeta e misturado com mesmo volume de tampãoacetato com solução corante 2,6-dicloroindofenol (Neon) e as absorbâncias lidas no comprimento de onda de $520 \mathrm{~nm}$. Na curva padrão de ácido ascórbico foram determinadas as concentrações de ácido ascórbico presentes nas adrenais e o conteúdo de ácido ascórbico da adrenal (AAA) foram padronizados e expressos em $\mu \mathrm{g} / 100 \mathrm{mg}$ adrenal $/ 100 \mathrm{~g}$ peso corpóreo para fins de comparação entre os ratos do grupo $\mathrm{G} 1$ tratados com salina e os ratos experimentais tratados com romã. Assim, o AAA expressou um índice que permitiu mensurar o nível de estresse dos animais em resposta ao processo inflamatório induzido e de avaliar a hipótese do efeito adaptógeno atenuador de estresse da romã.

Foto 1 - Rato após a indução do processo inflamatório.

Fonte: Produção própria dos autores do estudo.
Foto 2 - Rato do grupo G2 sendo tratado com gotas de chá de romã no ferimento.

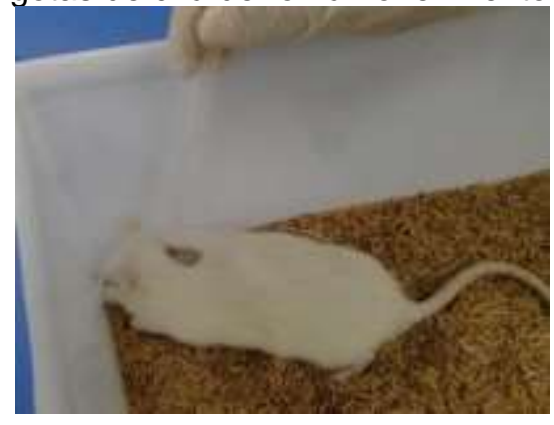

Fonte: Produção própria dos autores do octurno 


\subsection{ANÁLISES DE DADOS}

O teste paramétrico de escolha foi ANOVA inteiramente casualizado com diferente número de repetições, seguido pelo de Tukey para comparações múltiplas e o nível de significância foi fixado em $5 \%$ (BioEstat-5.3). Desse modo, foram consideradas significativas as diferenças entre as médias amostrais que apresentaram probabilidades de ocorrência menores que 0,05.

\section{RESULTADOS E DISCUSSAOO}

Os resultados obtidos dos parâmetros de interesse descritos na avaliação do estresse em ratos do grupo G1-controle (salina) e dos grupos experimentais (G2 = chá, G3 = polpa e G4 = chá+polpa) encontram-se na Tabela 1. Os pesos das adrenais estão expressos em miligramas (mg) e a concentração de ácido ascórbico da adrenal foi obtido na curva padrão e depois normalizado para $100 \mathrm{mg}$ de peso da adrenal e por grama de peso corporal individual dos ratos. Esse parâmetro foi designado como conteúdo de ácido ascórbico da adrenal (AAA) e expresso em $\mu \mathrm{g} / 100 \mathrm{mg} / \mathrm{g}$ de peso corporal.

Tabela 1 - Médias \pm desvio padrão de: Peso da adrenal $(P, m g)$ e conteúdo de ácido ascórbico da adrenal (AAA, $\mu \mathrm{g} / 100 \mathrm{mg} / \mathrm{g}$ ) de ratos do grupo G1-controle (salina 0,9 \%); grupo G2 (chá de romã); grupo G3 (polpa de romã) e grupo G4 (chá + polpa de romã), UnirG, Gurupi, TO, 2017.

\begin{tabular}{cccccccc}
\hline \multicolumn{2}{c}{ G1 } & \multicolumn{2}{c}{ G2 } & \multicolumn{2}{c}{ G3 } & \multicolumn{2}{c}{ G4 } \\
$\mathbf{P}$ & AAA & P & AAA & P & AAA & P & AAA \\
\hline 43,4 & 11,7 & 33,7 & 31,48 & 38,1 & 20,74 & 31,71 & 21,82 \\
6,1 & 7,8 & 7,9 & 16,41 & 11,2 & 5,23 & 8,63 & 9,24 \\
\hline
\end{tabular}

Fonte: Produção própria dos autores do estudo.

A Figura 1 mostra o gráfico de absorbância versus concentração de ácido ascórbico (quadruplicatas), que representou a curva padrão média utilizado para calcular os valores de conteúdo de AAA a partir das absorbâncias obtidas nas amostras. 


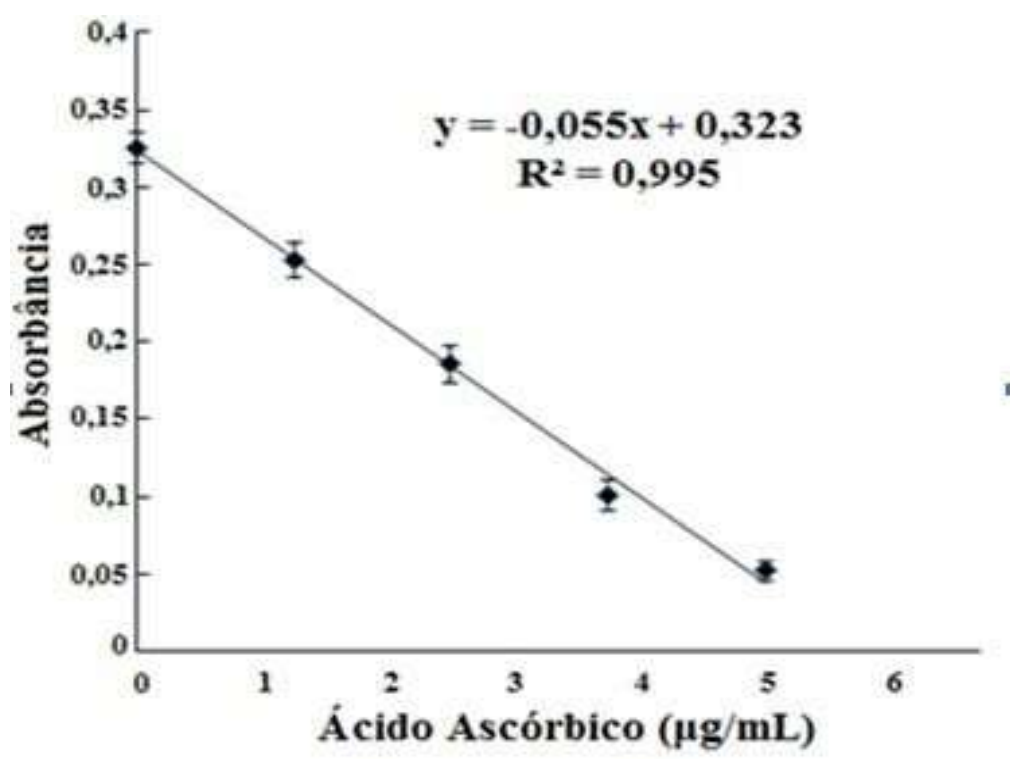

Figura 1 - Curva média obtida de absorbância versus concentração de ácido ascórbico. As absorbâncias obtidas das amostras foram inseridas na reta e determinado o conteúdo de ácido ascórbico da adrenal (AAA). A reta de regressão linear mostra um grau de ajuste dos pontos de 99,5\% (valor de $\mathrm{R}^{2}$ ).

A depleção de AAA observada nos ratos do grupo G1-controle (tratados com salina) indica relevante grau de estresse (ARAD et al.,1985; CONTARTEZE et al., 2007). A análise estatística confrontando as médias de AAA entre os grupos, sugere que a romã promoveu uma ação anti-estressora $(F=5,8 ; p=0,005)$. Portanto, há evidências de que a romã possa atenuar o efeito estressor decorrente do processo inflamatório induzido pelas feridas, caracterizando-se como uma planta medicinal candidata a ser classificada possuindo propriedade adaptógena. Estudos histológicos e morfológicos realizados por Pirbalouti et al (2010) mostram que houve redução do tamanho e o formato da ferida precocemente e aumentou o volume de colágeno em ratos tratados com o extrato de Punica granatum, comparado ao do grupo controle. Apesar da utilização de metodologias diferentes, ambos ratificam a atuação da romã na atenuação do processo inflamatório.

A diminuição do peso da adrenal sugere a ocorrência de uma atrofia da mesma ( $F=$ $5,9 ; p<0,01)$ nos animais tratados com romã, o que corrobora o resultado do aumento verificado no conteúdo de $A A A$, possivelmente pela queda sérica de corticosterona (principal glicocorticoide liberado pelo córtex das adrenais de roedores), confirmando uma resposta anti-estresse promovida pela romã na diminuição da atividade de feedback no eixo hipotálamo-hipófise-adrenal (HHA) (ANDERSEN et al., 2004; MOSTL et al., 2002; DALLMAN, 1993). A atrofia das adrenais poderia ser explicada pela diminuição no nível 
sérico do hormônio adrenocorticotrófico (ACTH) e, assim, diminuindo a intensidade da ação trófica estimulante deste hormônio na região córtico-adrenal promotora do processo de liberação de hormônios envolvidos no estresse (glicocorticoides, mineralocorticoides e adrenalina).

A elevação de AAA, em resposta a ação anti-estresse promovida pela romã no eixo HHA, atua em sinergismo com o efeito anti-estresse oxidativo propiciado pelo fortalecimento do sistema de defesa enzimático sugerido em estudos feitos por Haiyan Yan et al (2015). Vinculado ao tema, observou-se em estudo envolvendo ratos com colite ulcerativa induzido por ácido acético, a ação do ácido L-ascórbico como agente redutor de radicais livres advindos do dano oxidativo de lipídeos e de lipoproteínas durante a progressão da colite ulcerativa e como antioxidante nos fluidos extracelulares, segundo Zerín et al (2010).

Quanto as abordagens experimentais nos estudos dos aspectos relacionados a permeabilidade capilar e da quantidade de exsudato das feridas induzidas nos ratos do grupo G1 tratados com salina e dos grupos tratados com romã (chá, polpa e chá+polpa), os resultados obtidos estão apresentados nas Tabelas 2 e 3.

Tabela 2 - Médias \pm desvio padrão do peso relativo de exsudato (em \%) das feridas cicatrizadas em ratos do grupo controle-G1 (salina 0,9 \%); G2 (chá de romã); G3 (polpa de romã); G4 (chá+polpa de romã), UnirG, Gurupi, TO, 2017.

\begin{tabular}{cccc}
\hline G1 - Controle & G2 & G3 & G4 \\
\hline 70,38 & 68,85 & 64,32 & 75,47 \\
\hline 6,39 & 5,52 & 6,24 & 8,73 \\
\hline
\end{tabular}

Fonte: Produção própria dos autores do estudo.

Tabela 3 - Médias \pm desvio padrão do Índice de Permeabilidade Vascular do azul de Evans (IPV) padronizada em $\mu \mathrm{g} / \mathrm{g}$ de tecido das feridas cicatrizadas em ratos do grupo G1-controle (salina 0,9 \%); G2 (chá de romã); G3 (polpa de romã); G4 (chá+polpa de romã), UnirG, Gurupi, TO, 2017.

\begin{tabular}{cccc}
\hline G1 - Controle & G2 & G3 & G4 \\
\hline 62,8 & 12,8 & 20,8 & 19,7 \\
\hline 28,7 & 3,4 & 8,3 & 9,6 \\
\hline
\end{tabular}

Fonte: Produção própria dos autores do estudo.

O Índice de Permeabilidade Vascular (IPV) ao azul de Evans (AE), expresso em $\mu \mathrm{g} / \mathrm{g}$ de tecido das feridas cicatrizadas, foi determinado interpolando os dados de absorbância 
das amostras na curva padrão de $\mathrm{AE}$ apresentado na Figura 2, onde o coeficiente de regressão linear é de $99,4 \%$ o que outorga resultados fidedignos obtidos de IPV.

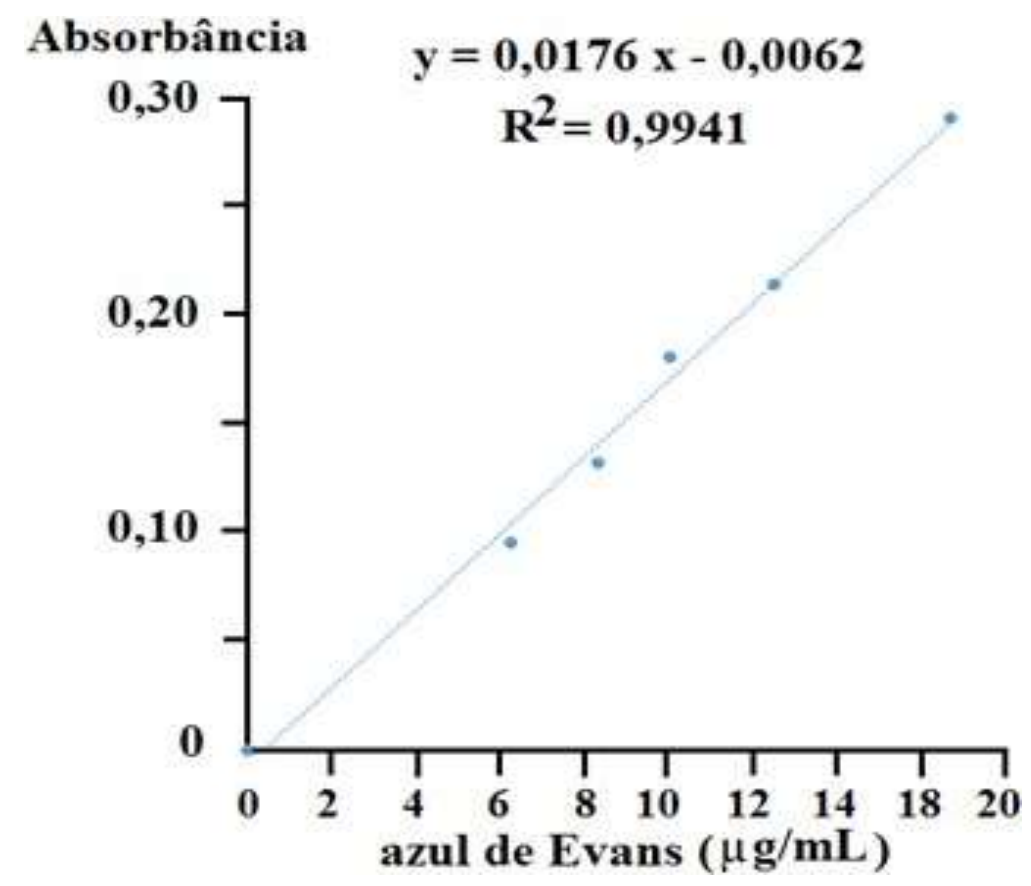

Fonte: Produção própria dos autores do estudo.

Figura 2 - Curva padrão de azul de Evans. Coeficiente de regressão linear mostra um grau de ajuste dos pontos à reta de $99,4 \%$.

Para a análise de dados do conteúdo de exsudato das feridas, os resultados da Tabela 2 não mostrou haver significância $(F=2,7 ; P=0,0677)$ entre as médias obtidas, ou seja, o conteúdo de exsudato foi igual em todas as condições de tratamento delineadas para este trabalho, embora a ANOVA aplicada aos dados de IPV ao AE mostre uma significância estatística ao nível de 5\%, cujas médias encontram-se na Tabela 3 ( $F=9,8 ; P$ $=0,0011)$.

A significância estatística encontrada nos dados de IPV ao AE, corrobora os dados obtidos por Maia et al (2015) que, com o método da nebulização pulmonar de romã, observaram uma redução da resposta inflamatória pulmonar em fêmeas de ratos da espécie Rattus norvegicus.

O azul de Evans, também conhecido como T-1824, é uma substância de peso molecular 980,809 Dalton que, se inoculada intravenosamente, passa a interagir com as albuminas plasmáticas pelos quais possuem uma forte afinidade (FREEDMAN e JOHNSON, 1969). Assim, cerca de $95 \%$ do $A E$ permanece estanque no sistema circulatório e, em condições normais, não extravasa para o interstício tissular através dos 
poros do endotélio capilar, os quais oferecem alta resistência à passagem das albuminas plasmáticas que possuem peso molecular de 65000 Dalton. Em condições fisiopatológicas de processos inflamatórios, no entanto, há intenso fluxo de exsudato do compartimento plasmático para o interstício através das paredes capilares, devido à pressão oncótica exercida pelas albuminas que carregam junto interligadas o AE (STEELE e WILHELM, 1966).

Considerando que o aumento da permeabilidade para as macromoléculas através dos fenestras capilares ocorra em fases iniciais dos processos inflamatórios (GEHLEN et al., 2004), pode-se observar que a quantidade de $A E$ é maior nos ratos do grupo G1 (controle), uma vez que estes foram tratados com salina, um fluido inócuo (Tabela 3). A diminuição da IPV ao AE ligadas às macromoléculas verificadas nos grupos G2, G3 e G4, ou seja, em ratos tratados com romã, evidencia, portanto, uma ação anti-inflamatória efetiva da romã em feridas abertas na região dorso costal de ratos.

\section{azul de Evans \\ $\mu \mathrm{g} / \mathrm{g}$ tecido}

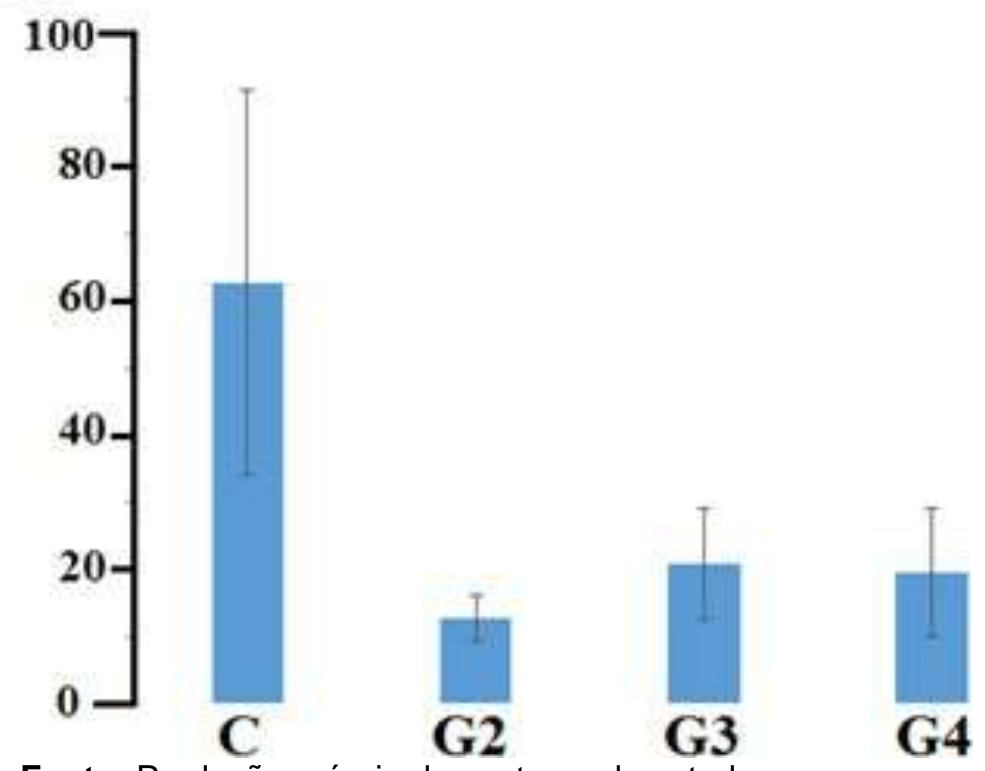

Fonte: Produção própria dos autores do estudo.

Figura 3 - Quantidade extravasada tecidual de azul de Evans nas feridas cicatrizadas retiradas de ratos do grupo controle-G1 (C), G2, G3 e G4, tratados respectivamente com salina (0,9\%), chá de romã, polpa de romã e chá+polpa de romã. UnirG, Gurupi, TO, 2017.

A Figura 3 ilustra os dados apresentados na Tabela 3 apenas para uma melhor visualização, assim como para avaliar a magnitude da variabilidade, acuracidade e precisão da técnica por inoculação de AE via portal hepática para a mensuração dos IPV em vasos lesados que foi utilizado neste trabalho. 
A análise estatística dos dados da Tabela 2, ou seja, referentes aos pesos dos exsudatos, em \% relativa, nas feridas cicatrizadas em ratos do grupo $\mathrm{G} 1$ comparados aos que foram obtidos nos grupos G2, G3 e G4, não mostrou haver diferenças nas médias obtidas, um resultado que não acompanhou o que era esperado perante os obtidos com os IPV ao AE, assim como não corrobora com o sinergismo (Local + Sistêmico) dos efeitos atenuantes observados no estudo histopatológico de Nascimento Júnior et al (2016). Uma possível explicação para este resultado pode ser atribuída à manipulação e/ou à pesagem dos tecidos, o qual apesar de usar balança analítica, quando comparado à espectrofotometria é de longe menor quanto à sua acuracidade e precisão. Decorrente disso, o teste não conseguiu detectar uma diferença que poderia ser considerada significativa, embora o valor de $\mathrm{P}=0,0677$ tenha ficado próximo do nível de significância estabelecido para o teste $(P=0,05)$, fazendo-se necessário, por conseguinte, aumentar o tamanho amostral para que uma diferença mínima possa ser detectada pelo teste estatístico.

\section{CONSIDERAÇÓES FINAIS}

Fundamentado no conjunto de evidências obtidas e apresentadas, pode-se concluir que a romã, nas suas múltiplas formas de apresentação, possui ação anti-inflamatória em feridas de pele, além de atenuar a resposta de estresse às lesões induzidas, sendo, portanto, uma planta medicinal candidata a ser classificada como fitoterápica e com propriedades adaptógenas.

\section{REFERÉNCIAS}

AHMEDA, H. H; EL-ABHAR, H. S; HASSANINC, E. A. K; ABDELKADER, N. F; SHALABY, M. B. Punica granatum suppresses colon cancer through downregulation of Wnt/-Catenin in rat model. Revista Brasileira de Farmacognosia, v.27, p. 627-635, 2017. Disponível em: <https://www.sciencedirect.com/science/article/pii/S0102695X17301667>.

ANDERSEN, M.L., BIGNOTTO, M., MACHADO, R.B., TUFIK, S. Different stress modalities result in distinct steroid hormone responses by male rats. Brazilian Journal of Medical and Biological Research, Ribeirão Preto, v.37 n.6, p.791-7, 2004. Disponível em: <http://www.scielo.br/scielo.php?script=sci_arttext\&pid=S0100-879X2004000600003>.

ARAD, I; SIDI, A; SHOHAMI, E. Effect of acute hypoxia on ascorbate content of plasma, cerebral cortex, and adrenal gland. J Neurochem, v.45, n3, p. 766- 769, 1985.

ARGENTA, J. A.; PASQUAL, M.; PEREIRA, C. V.; DIAS, D.R; BARBOSA, R. A.; PEREIRA, L. J. Efeito do extrato de romã (Punica granatum) sobre bactérias cariogênicas: estudo in 
DOI: DOI: 10.18605/2175-7275/cereus.v11n3p112-122 Revista Cereus

2019, vol. 11, no 3
Marcelino, K. B; Silva Júnior, N. A.; Freitas, A. C. G; Campus, C. C; Ogawa, W. N. Efeito da Punica Granatum (romã) em processos inflamatórios induzidos em ratos e suas implicações no eixo hipotálamo-hipófise-adrenal.

vitro e in vivo. Arquivos em Odontologia, Belo Horizonte, v.48 n.4, p. 218-226, 2012. Disponível em: <https://seer.ufmg.br/index.php/arquivosemodontologia/article/viewFile/2031/1469>.

CARLINI, E.A; MENDES, F.R. Protocolos em Psicofarmacologia Comportamental: Um Guia Para a Pesquisa de Drogas com ação sobre o SNC, com ênfase nas plantas medicinais. São Paulo, ed. Fap-Unifesp, p.323-354, 2011.

CONTARTEZE, R.V.L; MANCHADO, F.B.; GOBATTO, C.A.; ROSTOM DE MELO, M.A. Biomarkers of stress in rats exercised in swimming at intensities equal and superior to the maximal estable lactate phase. Revista Brasileira de Medicina do Esporte, v.13, n.3, p.150154, 2007.

DALLMAN, M.F. Stress update: adaptation of the hypothalamic-pituitary-adrenal axis to chronic stress. Trends End Metabol, v.4, p.62-69, 1993.

DAVYDOV, M.; KRIKORIAN A.D. Eleutherococcus senticosus (Rupr. \& Maxim.) Maxim. (Araliaceae) as an adaptogen: a closer look. Journal of Ethnopharmacology, v.72, p.345393, 2000.

DEGASPARI, C. H; DUTRA, A. P. C. Propriedades Fitoterápicas da Romã (Punica granatum L.). Revista Visão Acadêmica, Cuiritiba, v.12, n.1, p.36-46, 2011. Disponível em: $<$ http://revistas.ufpr.br/academica/article/view/27237/18143>.

FERREIRA, V. F.; PINTO, A. C. A fitoterapia no mundo atual. Química Nova, São Paulo, v.33, n. 9, p.1829, 2010. Disponível em: < http://www.scielo.br/pdf/qn/v33n9/01.pdf>

FREEDMAN, F.B., JOHNSON, J. Equilibrium and kinetic properties of the Evans bluealbumin system. Am. J. Physiol, Minneapolis, v.16, p.675-81, 1969. Disponível em: $<$ https://www.physiology.org/doi/abs/10.1152/ajplegacy.1969.216.3.675?journalCode=ajpl egacys.

GEHLEN, M.L.; MOREIRA, H.; MOREIRA, L.; SABAG, F.P.; REPKA, J.C.D. Avaliação espectrofotométrica do azul de Evans na reação inflamatória da córnea: estudo experimental em coelhos. Arquivos. Brasileiros de Oftalmologia, São Paulo, v.67, p.219-25, 2004. Disponível em: <http://www.scielo.br/scielo.php?script=sci_arttext\&pid=S0004$27492004000200007 \&$ Ing $=$ pt\&nrm=iso\&tlng=pt>.

MAIA FILHO, A. L. M; NASCIMENTO, W. M; BARROS, E. M. L.; COSTA, D. A. F.; ALMEIDA, M. K. C. Efeito anti-inflamatório da romã (punica granatum) na síndrome do desconforto respiratório agudo. Revista Saúde em Foco, Teresina, v.2 , n.2, 2015. Disponível em:

<http://www4.fsanet.com.br/revista/index.php/saudeemfoco/article/view/841/874>.

MÖSTL, E.; PALME R. Hormones as indicators of stress. Domest. Anim. Endocrinol. v.23, p.67-74, 2002.

NASCIMENTO JÚNIOR, B J; SANTOS, A.M.T.; SOUZA, A.T.; SANTOS, E.O.; XAVIER, M.R.; MENDES,R.L.; AMORIM, E.L.C. Estudo da ação da romã (Punica granatum L.) na cicatrização de úlceras induzidas por queimadura em dorso de língua de ratos Wistar 
(Rattus norvegicus). Revista Brasileira de Plantas Medicinais, v.18 (2), p.423-432, 2016. Disponível em: <http://www.scielo.br/pdf/rbpm/v18n2/1516-0572-rbpm-18-2-0423.pdf>.

PIRBALOUTI, A. G; KOOHPAYEH, A; KARIMI, I. The wound healing activity of flower extracts of punica granatum and achillea kellalensis in wistar rats. Acta Poloniae Pharmaceutica, v.67, n.1, p.107-110, 2010. Disponível em: <http://ptfarm.pl/pub/File/acta_pol_2010/1_2010/107-110.pdf>.

SANTOS, M.P.; SANTOS, C. C.; CARVALHO, M. P. Cannabis sativa e Salvia divinorum uso irresponsável de plantas medicinais com atividade psicoativas. Revista de Trabalhos Acadêmicos, n. 2, p.1-7, 2011.

STEELE, R. H.; WILHELM, D. L. The inflammatory reaction in chemical injury. Increased vascular permeability and erythema induced by various chemicals. Br. J. Exp. Pathol., v.47, p.612-23, 1966.

TAKATA, W.; SILVA, E. G.; CORATO, J. M.; FERREIRA, G. Germinação de sementes de romäzeiras (Punica granatum L.) de acordo com a concentração de giberelina. Revista Brasileira de Fruticultura, Jaboticabal, v. 36, n. 1, p. 254-260, 2014. Disponível em: < http://www.scielo.br/pdf/rbf/v36n1/v36n1a31.pdf>.

TOZETTO, J. T.; TOZETTO, A. T.; HOSHINO, B. T.; ANDRIGHETTI, C. R. Extract of punica granatum I.: an alternative to bht as an antioxidant in semissolid emulsified systems. Química Nova, São Paulo, v.40, n.1, p.97-104, 2017. Disponível em: <http://www.scielo.br/pdf/qn/v40n1/0100-4042-qn-40-01-0097.pdf>.

TRINDADE, M. P.; FONSECA, L.; JUIZ, P.J. L. Atividade antimicrobiana da tintura da casca de romã (Punica granatum) sobre cepas de Staphylococcus aureus e Streptococcus pyogenes: estudo in vitro. Revista Brasileira de Pesquisa em Saúde, v.11, n.4, p.49-54, 2009. Disponível em:<http://periodicos.ufes.br/RBPS/article/viewFile/355/266>.

YAN, H.; WANG, H.; ZHANG, X.; et al. Ascorbic acid ameliorates oxidative stress and inflammation in dextran sulfate sodium-induced ulcerative colitis in mice.

Int. J. Clin. Exp. Med., v.8, n.11, 2015. Disponível em: <https://www.ncbi.nlm.nih.gov/pmc/articles/PMC4723782/>.

ZERIN, M.; KARAKILÇIK, A. Z.; BITIREN, M.; MUSA, D.; ÖZGÖNÜL, A.; SELEK, S.; NAZLIGÜL, Y.; UZUNKÖY, A. Vitamin C modulates oxidative stress-induced colitis in rats. Turkish Journal of Medical Sciences, v.40, n.6, p.871-879, 2010. Disponível em: <http://dergipark.gov.tr/download/article-file/128734>. 